\title{
Landmark estimation of transition probabilities in non-Markov multi-state models with covariates
}

\author{
Rune Hoff ${ }^{1}$ • Hein Putter ${ }^{3}$ • Ingrid \\ Sivesind Mehlum ${ }^{2}$. Jon Michael Gran ${ }^{1}$
}

Received: date / Accepted: date

\begin{abstract}
In non-Markov multi-state models, the traditional Aalen-Johansen (AJ) estimator for state transition probabilities is generally not valid. An alternative, suggested by Putter and Spitioni, is to analyse a subsample of the full data, consisting of the individuals present in a specific state at a given landmark time-point. The AJ estimator of occupation probabilities is then applied to the landmark subsample. Exploiting the result by Datta and Satten, that the AJ estimator is consistent for state occupation probabilities even in non-Markov models given that censoring is independent of state occupancy and times of transition between states, the Landmark Aalen-Johansen (LMAJ) estimator provides consistent estimates of transition probabilities. So far, this approach has only been studied for non-parametric estimation without covariates. In this paper, we show how semi-parametric regression models and inverse probability weights can be used in combination with the LMAJ estimator to perform covariate adjusted analyses. The methods are illustrated by a simulation study and an application to population-wide registry data on work, education and health-related absence in Norway. Results using the traditional AJ estimator and the LMAJ estimator are compared, and show large differences in estimated transition probabilities for highly non-Markov multi-state models.
\end{abstract}

Keywords Multi-state models · the Markov property · transition probabilities $\cdot$ landmarking $\cdot$ Aalen-Johansen estimator

Rune Hoff

E-mail: rune.hoff@medisin.uio.no

1 Oslo Center for Biostatistics and Epidemiology, University of Oslo and Oslo University Hospital, Norway

2 National Institute of Occupational Health, Oslo, Norway

3 Department of Medical Statistics and Bioinformatics, Leiden University Medical Center, Leiden, The Netherlands 


\section{Introduction}

Multi-state models, as an extension of traditional time-to-event models, have received increasing amount of attention over the last decades. See for example tutorials by Hougaard (1999), Andersen and Keiding (2002), Putter et al (2007), Meira-Machado et al (2008) and Andersen and Pohar Perme (2013) or the mstate package in $\mathrm{R}$ (de Wreede et al, 2011). An attractive feature of multi-state models, is that traditional hazard-based methods from survival analysis can be applied to estimate transition intensities. This includes the Nelson-Aalen estimator for the cumulative hazard and any hazard regression model, such as the Cox proportional hazards model and Aalen's additive hazards model.

In addition to transition intensities, two other quantities are often of interest when making inference from multi-state models; state transition probabilities and state occupation probabilities. When the specified multi-state model is Markov, both transition probabilities and occupation probabilities can be consistently estimated in a simple manner by plugging the transition intensities into the so-called matrix product-integral formula for the empirical transition matrix, often denoted the Aalen-Johansen estimator (Aalen et al, 2008). For multi-state models that are not Markov, Datta and Satten (2001), in their seminal paper, showed that state occupation probabilities still are consistently estimated using the product-integral formula, given that censoring is independent of states occupancy and transition times between states. However, this is not the case for state transition probabilities.

Explicit formulas for transition probabilities when multi-state models are not Markov are available for some types of models, for example for semiMarkov multi-state models without loops (Andersen and Pohar Perme, 2008; Titman, 2015). There is also currently a rich ongoing methodological development on methods for estimating state transition probabilities in more general semi- and non-Markov multi-state models (Allignol et al, 2014; Titman, 2015; de Uña-Álvarez and Meira-Machado, 2015; Putter and Spitoni, 2018). While the Aalen-Johansen estimates make use of the full data set, which is made possible by the Markov assumption, the methods from these papers are all based on the idea of subsampling. Most recently, Putter and Spitoni (2018) suggested the analysis of a subset of the population, based on the individuals being in a specific state at a specific time point, which is a form of landmarking. Within the landmark subset, they argue that the Aalen-Johansen (AJ) estimator can be applied to obtain consistent estimates of state transition probabilities. This holds because transition probabilities can be seen as occupation probabilities in specific landmark subsets. Thus, due to the result of Datta and Satten, the product-integral formula can be used to consistently estimate transition probabilities after subsampling on a landmark. Putter and Spitioni's estimator, which they named the Landmark Aalen-Johansen (LMAJ) estimator, is shown to be more efficient than alternative methods and is applicable in arbitrary multi-state models (Putter and Spitoni, 2018). 
Note that the LMAJ estimator was introduced for a setting with unadjusted non-parametric estimation of transition intensities and that the result of Datta and Satten also was proven in the case without covariates. However, there are many applications where it is desirable to include, or adjust for, covariate information when estimating state transition probabilities, e.g. using Cox regression or additive hazards regression for modelling the transition intensities and by inverse probability of treatment (IPT) weighting (Robins et al, 2000). One could have a situation where the interest is in 1) the effect of a single binary randomised treatment variable on the transition probabilities, 2) adjusting for confounding variables to identify the effect of a binary non-randomised treatment on transition probabilities, or 3) covariate-specific prediction of transition probabilities. To our knowledge, the validity of the Datta and Satten result, or the landmark approach by Putter and Spitioni, has not been studied for models with covariates.

In this paper, we argue for the validity of the result in Datta and Satten (2001) in two different settings; one using covariate adjusted transition intensities based on hazard regression models and one where such intensities are estimated after IPT weighting. As a result, we argue that the LMAJ estimator of Putter and Spitioni also can be used to consistently estimate covariate adjusted or IPT weighted state transition probabilities. We study the properties of the methods in a simulation study and illustrate them through an application to Norwegian registry data on work, education and health-related absence.

\section{The landmark approach to estimating transition probabilities}

\subsection{Multi-state models}

A multi-state model is a model for a stochastic process $\{X(t) \mid t \geq 0\}$, taking values in the state space $\mathcal{S}=\{1,2,3, \ldots, S\}$. The model is defined by the transition intensities

$$
\alpha_{j k}\left(t, \mathcal{H}_{t-}\right)=\lim _{\Delta t \rightarrow 0} \frac{\operatorname{Pr}\left(X(t+\Delta t)=k \mid X(t-)=j, \mathcal{H}_{t-}\right)}{\Delta t}, \quad \forall j, k \in \mathcal{S}: j \neq k
$$

where $\mathcal{H}_{t-}$ is the history of the process up to just before time $t$. If we assume that the process is Markov, then $\alpha_{j k}\left(t, \mathcal{H}_{t-}\right)=\alpha_{j k}(t)$.

Let $\boldsymbol{P}(s, t)$ be the $S \times S$ transition probability matrix with elements $P_{j k}(s, t)$, for all $j$ and $k$ from 1 to $S$, where

$$
P_{j k}(s, t)=\operatorname{Pr}\left(X(t)=k \mid X(s)=j, \mathcal{H}_{s}\right),
$$

for two time points $s$ and $t$, where $s \leq t$.

When the multi-state model is Markov, the state transition probabilities, now $P_{j k}(s, t)=\operatorname{Pr}(X(t)=k \mid X(s)=j)$, are given by the formula

$$
\boldsymbol{P}(s, t)=\pi_{(s, t]}(\mathbf{I}+d \boldsymbol{A}(u)),
$$


where $\boldsymbol{A}(t)$ is the matrix of cumulative transition intensities with elements $A_{j k}(t)=\int_{0}^{t} \alpha_{j k}(s) d s$ for $j \neq k$ and $A_{j j}(t)=-\sum_{k \neq j} A_{j k}(t)$. See for example Andersen and Pohar Perme (2013) for more. Let then $\widehat{\boldsymbol{A}}(t)$ be the $S \times S$ matrix of estimated cumulative transition intensities, with elements $\widehat{A}_{j k}(t)$, $\forall j, k \in \mathcal{S}$. Given that $\widehat{\boldsymbol{A}}(t)$ is step function, we may then estimate the transition probabilities by the finite matrix product

$$
\widehat{\boldsymbol{P}}(s, t)=\prod_{(s, t]}(\mathbf{I}+d \widehat{\boldsymbol{A}}(u))=\prod_{(s, t]}(\mathbf{I}+\Delta \widehat{\boldsymbol{A}}(u)),
$$

where $\Delta \widehat{\boldsymbol{A}}(t)$ is the increment of $\widehat{\boldsymbol{A}}$ at time $t$. The state occupation probabilities $\boldsymbol{\pi}(t)$, a row vector with elements $\pi_{j}(t)=\operatorname{Pr}(X(t)=j)$, for all $j \in \mathcal{S}$, can then be calculated by

$$
\widehat{\boldsymbol{\pi}}(t)=\boldsymbol{\pi}(0) \widehat{\boldsymbol{P}}(0, t)=\boldsymbol{\pi}(0) \prod_{(0, t]}(\mathbf{I}+\Delta \widehat{\boldsymbol{A}}(u)),
$$

where $\boldsymbol{\pi}(0)$ is assumed known and corresponds to the initial state distribution at time zero.

Without covariates, the elements of $\boldsymbol{A}(t)$ can be estimated by the NelsonAalen estimator. In settings with a vector of transition specific covariates $\boldsymbol{z}_{j k}$, corresponding cumulative transition intensities $A_{j k}\left(t \mid \boldsymbol{z}_{j k}\right)=\int_{0}^{t} \alpha_{j k}(u \mid$ $\left.\boldsymbol{z}_{j k}\right) d u$, can be estimated using traditional hazard regression models. This includes the Cox proportional hazards model paired with the Breslow estimator for the cumulative baseline hazard (Breslow, 1972), and the additive hazards model (Aalen et al, 2008).

\subsection{The Datta-Satten result}

Datta and Satten (2001) showed that the matrix product-integral formula in (1) produce consistent estimates of the state occupation probabilities even when the underlying multi-state process is not Markov, given that censoring is independent of state occupancy and times of transition between states. Note that this censoring assumption is a stronger assumption than the common assumption of independent censoring (Aalen et al, 2008, p. 123). However, when such censoring dependencies occur, it was later showed in Datta and Satten (2002) that valid estimates of the state occupation probabilities still could be obtained by introducing inverse probability of censoring (IPC) weights to the formula in (1). For more on proofs, asymptotics and inference for the results in Datta and Satten (2001), see also Glidden (2002). Among other things, Glidden showed that the Greenwood estimates of the standard errors for occupation probabilities in the case without covariates also are valid without the Markov assumption. See e.g. Andersen et al (1993, p. 290 - 295), for more on these estimators. We now argue for the validity of the Datta-Satten result in multi-state models based on hazard regression models (Section 2.2.1) and in inverse probability of treatment weighted multi-state models (Section 2.2.2). 
2.2.1 Validity of the Datta-Satten result in multi-state models based on hazard regression models

Consider a situation where we want to estimate covariate specific state occupation probabilities. With baseline covariates $\boldsymbol{z}_{j k}$, intensities $\alpha_{j k}\left(t \mid \boldsymbol{z}_{j k}\right)$ can be modelled by any hazard regression model from traditional event history analysis. Let us for simplicity consider the situation where the same covariates are used for all transitions, so that $\boldsymbol{z}_{j k}=\boldsymbol{z}$, even though this limitation is not necessary.

Covariate specific transition intensities in Cox proportional hazards models from state $j$ to state $k$ takes the form

$$
\alpha_{j k}(t \mid \boldsymbol{z})=\alpha_{j k 0}(t) \exp \left(\boldsymbol{\beta}_{j k}^{T} \boldsymbol{z}\right) .
$$

Here, $\boldsymbol{\beta}_{j k}$ is a vector of regression coefficients and $\alpha_{j k 0}(t)$ is the baseline intensity for transitions from state $j$ to state $k$. The cumulative baseline transition intensities $A_{j k 0}(t)=\int_{0}^{t} \alpha_{j k 0}(u) d u$ can be estimated using the Breslow estimator

$$
\widehat{A}_{j k 0}(t)=\sum_{T_{i} \leq t} \frac{\Delta \bar{N}_{j k}\left(T_{i}\right)}{\sum_{l} Y_{l}\left(T_{i}\right) \exp \left(\hat{\boldsymbol{\beta}}_{j k}^{T} \boldsymbol{z}_{l}\right)}, j \neq k
$$

where $T_{i}$ are the ordered times when transitions occurs, $\Delta \bar{N}_{j k}(t)$ is the number of transitions observed from state $j$ to state $k$ at time $t$, and $Y_{l}(t)$ is an at risk indicator that takes the value 1 if individual $l$, with covariates $\boldsymbol{z}_{l}$, is observed to be in state $j$ just before time $t$. See for example Andersen et al (1993). Without covariates, this reduces to the Nelson-Aalen estimator. With covariates, the estimate for the cumulative transition intensity for a given covariate pattern $z$ is

$$
\widehat{A}_{j k}(t \mid \boldsymbol{z})=\widehat{A}_{j k 0}(t) \cdot \exp \left(\hat{\boldsymbol{\beta}}_{j k}^{T} \boldsymbol{z}\right), j \neq k
$$

Alternatively, we can model transition intensities using the additive hazards model for each transition $j \rightarrow k$ (Aalen et al, 2001), with

$$
\alpha_{j k}(t \mid \boldsymbol{z})=\beta_{j k 0}(t)+\boldsymbol{\beta}_{j k}^{T}(t) \boldsymbol{z},
$$

where $\boldsymbol{\beta}_{j k}^{T}(t)$ are time-varying regression functions and $\beta_{j k 0}(t)$ is the baseline $j \rightarrow k$ transition intensity. The cumulative regression functions:

$$
\boldsymbol{B}_{j k}(t)=\left[\int_{0}^{t} \beta_{j k 0}(u) d u, \int_{0}^{t} \boldsymbol{\beta}_{j k}^{T}(u) d u\right]^{T},
$$

can easily be estimated using regular least squares techniques and the resulting estimate $\widehat{\boldsymbol{B}}_{j k}(t)$ corresponds to a multivariate Nelson-Aalen estimator. If $\boldsymbol{z}$ is restricted to a binary covariate only, with element $z=0$ or 1 , then $\widehat{B}_{j k 0}(t)$ is the Nelson-Aalen estimator for the group with $z=0$, and the element $\widehat{B}_{j k 1}(t)$ is the difference between the Nelson-Aalen estimators for the two groups. For a given covariate pattern $\boldsymbol{z}$, we may introduce $\boldsymbol{z}^{*}=\left(1, \boldsymbol{z}^{T}\right)^{T}$ and estimate the corresponding cumulative transition intensity by $\widehat{A}_{j k}(t \mid \boldsymbol{z})=\widehat{\boldsymbol{B}}_{j k}^{T}(t) \boldsymbol{z}^{*}$. 
In both cases, as long as the model assumptions of the selected hazard regression model hold, the validity of the formula in (1) should be preserved, and one can write

$$
\widehat{\boldsymbol{\pi}}(t \mid \boldsymbol{z})=\widehat{\boldsymbol{\pi}}(0 \mid \boldsymbol{z}) \prod_{(0, t]}(\mathbf{I}+d \widehat{\boldsymbol{A}}(u \mid \boldsymbol{z})),
$$

where $\widehat{\boldsymbol{\pi}}(0 \mid \boldsymbol{z})$ is estimated using, e.g., multinomial regression.

Formally one can argue for the validity of the Datta-Satten result in multistate models based on conditional intensities, using similar arguments as for non-conditional models. For example, inspired by the argument in Aalen et al (2001) for discrete time, we can consider the following justification of validity for the formula in (2) without any Markov assumption. For simplicity, we leave out censoring from this argument.

First, we note that

$$
\begin{aligned}
& \frac{1}{\Delta t}(\boldsymbol{\pi}(t+\Delta t \mid \boldsymbol{z})-\boldsymbol{\pi}(t \mid \boldsymbol{z}))=\frac{1}{\Delta t}\left(\left[\begin{array}{c}
\operatorname{Pr}(X(t+\Delta t)=1 \mid \boldsymbol{z}) \\
\vdots \\
\operatorname{Pr}(X(t+\Delta t)=S \mid \boldsymbol{z})
\end{array}\right]-\left[\begin{array}{c}
\operatorname{Pr}(X(t)=1 \mid \boldsymbol{z}) \\
\vdots \\
\operatorname{Pr}(X(t)=S \mid \boldsymbol{z})
\end{array}\right]\right)^{T} \\
& \quad=\frac{1}{\Delta t}\left(\left[\begin{array}{c}
\sum_{k=1}^{S} \operatorname{Pr}(X(t+\Delta t)=1, X(t)=k \mid \boldsymbol{z}) \\
\vdots \\
\sum_{k=1}^{S} \operatorname{Pr}(X(t+\Delta t)=S, X(t)=k \mid \boldsymbol{z})
\end{array}\right]-\left[\begin{array}{c}
\operatorname{Pr}(X(t)=1 \mid \boldsymbol{z}) \\
\vdots \\
\operatorname{Pr}(X(t)=S \mid \boldsymbol{z})
\end{array}\right]\right)^{T} \\
& =\boldsymbol{\pi}(t \mid \boldsymbol{z}) \cdot \frac{1}{\Delta t}(\boldsymbol{P}(t, t+\Delta t \mid \boldsymbol{z})-\boldsymbol{I}),
\end{aligned}
$$

for some time interval $(t, t+\Delta t]$.

Now, if $d \boldsymbol{A}(t \mid \boldsymbol{z})=\lim _{\Delta t \rightarrow 0+}(\boldsymbol{P}(t, t+\Delta t \mid \boldsymbol{z})-\boldsymbol{I})$, we get that

$$
\frac{d}{d t} \boldsymbol{\pi}(t \mid \boldsymbol{z})=\boldsymbol{\pi}(t-\mid \boldsymbol{z}) \cdot d \boldsymbol{A}(t \mid \boldsymbol{z}),
$$

which, by integrating on both sides, can be written as

$$
\boldsymbol{\pi}(t \mid \boldsymbol{z})=\boldsymbol{\pi}(0 \mid \boldsymbol{z})+\int_{0}^{t} \boldsymbol{\pi}(s-\mid \boldsymbol{z}) d \boldsymbol{A}(s \mid \boldsymbol{z}) .
$$

This integral equation has a unique solution after Volterra (1887) (Andersen et al, 1993, p. 90) given by

$$
\boldsymbol{\pi}(t \mid \boldsymbol{z})=\boldsymbol{\pi}(0 \mid \boldsymbol{z}) \overbrace{(0, t]}(\mathbf{I}+d \boldsymbol{A}(s \mid \boldsymbol{z})) .
$$

We recognise the above expression as the product-integral formula in (1). Note that the last part of the above argument has similarities with the proof of the product-integral formula for transition probabilities, revisited for example in Andersen and Pohar Perme (2013), p. 429, but that in our case, the proof does not involve any Markov assumption. In other words, we can identify 
$\boldsymbol{\pi}(t \mid \boldsymbol{z})$ without the Markov assumption if we have a consistent estimator for $\boldsymbol{A}(t \mid \boldsymbol{z})$. Following the above argument, standard errors can now be calculated plugging in the covariate specific covariance matrix in the formula in Andersen et al (1993), Eq. 4.4.17.

Note that we have not considered time-varying covariates, where $\boldsymbol{z}$ is replaced with some $\boldsymbol{z}(t)$. The use of external time-varying covariates could be a topic for future work, while the use of internal time-varying covariates is, as usual, expected to be problematic, see e.g. Aalen et al (2008). A weighted approach, as discussed in the following paragraphs, might then be the favourable approach.

\subsubsection{Validity of the Datta-Satten result in inverse probability of treatment weighted multi-state models}

Let us now consider a situation where we want to estimate the marginal effect of a single baseline covariate $a$, being an element in $\boldsymbol{z}$, for example a binary treatment variable with values 0 or 1 , on state occupation probabilities. The remaining covariates in $\boldsymbol{z}$, which we denote $\boldsymbol{c}$, can then be marginalised out using so-called propensity score or IPT weights (Rosenbaum, 1987; Robins et al, 2000). The weights are used to model a counterfactual scenario, where treatment assignment is no longer dependent on covariates, or in other words; a scenario where treatment is randomised. When such weights are estimated, e.g. using logistic regression, inference on the marginal effect of treatment can be performed by analysing the weighted data set.

If one accepts that the IPT weighting procedure simply is a change of probability measure from the observed measure to a randomised treatment measure (see for example Røysland (2011)), then the validity of the Datta-Satten result on IPT weighted multi-state models is trivial. One can also use a similar argument as in Datta and Satten (2002), who prove the validity of (1) without Markov assumptions, for IPC weighted estimates under dependent censoring. Here, censoring probabilities were calculated using the Aalen additive model. Other models for the censoring mechanism could also be used, for example pooled logistic regression as in Robins et al (2000).

Pointwise confidence intervals for occupation probabilities in weighted models can be estimated in the lines of Andersen et al (1993), p. 294, and Datta and Satten (2002). In the latter paper, they suggest using bootstrapping for simplicity. Another alternative is to use conservative robust sandwich estimates of the variance (Cole et al, 2007). For example, for Nelson-Aalen or Cox proportional hazards estimates of the transition intensities, the robust estimated covariance matrices from Lin and Wei (1989) can be plugged into the formula for the covariance matrix for state probabilities in Andersen et al (1993), Eq. 4.4.17. Similar robust sandwich type estimators for the covariance matrix can be used also for the additive hazards model, see for example Scheike (2002).

Given that the model for the treatment weights are correctly specified, IPT weighting will reduce to a regression model with a single, typically binary, covariate as discussed earlier. One can then also analyse the two subsets of the 
weighted data separately, for treatment equal to 0 and 1 . This will correspond to plugging weighted Nelson-Aalen estimates for the transition intensities into (1) in accordance with Datta and Satten (2001).

When the target of inference is the difference between two state occupation probabilities for state $j, \pi_{j}^{1}(t)$ and $\pi_{j}^{0}(t)$, for two disjoint groups 0 and 1 , the standard errors can be obtained by

$$
\mathrm{SE}\left[\widehat{\pi}_{j}^{1}(t)-\widehat{\pi}_{j}^{0}(t)\right]=\sqrt{\widehat{\operatorname{Var}}\left(\widehat{\pi}_{j}^{1}(t)\right)+\widehat{\operatorname{Var}}\left(\widehat{\pi}_{j}^{0}(t)\right)} .
$$

$\widehat{\operatorname{Var}}\left(\widehat{\pi}_{j}^{g}(t)\right)$ is the estimated variance of the estimated state occupation probabilities $\widehat{\pi}_{j}^{g}(t)$ for group $g(g=0,1)$. The estimated state occupation probabilities are given as

$$
\widehat{\pi}_{j}^{g}(t)=\sum_{i=1}^{S} \pi_{i}^{g}(0) \widehat{P}_{i j}^{g}(0, t),
$$

linear combination of the estimated transition probabilities. Thus the variance is had by

$$
\widehat{\operatorname{Var}}\left(\widehat{\pi}_{j}^{g}(t)\right)=\sum_{i=1}^{S} \sum_{h=1}^{S} \pi_{i}^{g}(0) \pi_{h}^{g}(0) \widehat{\operatorname{Cov}}\left(\widehat{P}_{i j}^{g}(0, t), \widehat{P}_{h j}^{g}(0, t)\right)
$$

The estimated covariances on the right side can be had from Greenwood-type formulas given by (4.4.17)-(4.4.20) in Andersen et al (1993). Alternatively, $\widehat{\operatorname{Var}}\left(\widehat{\pi}_{j}^{g}(t)\right)$ may be obtained with bootstrapping. Following the arguments from earlier, these variances could be estimated in a similar manner for covariate adjusted models, by instead plugging in estimates of the covariate specific covariance matrix in formula (4.4.17) in Andersen et al (1993), and for inverse probability weighted models by using robust estimators described above or by bootstrapping.

Note again that we only have considered baseline treatment and weights. For similar reasons as for the hazard regression models, if we have timedependent covariates, that is; a time-dependent treatment and/or confounders; analysis will be more involved. However, a convenient feature of multi-state models is that time-dependent treatment variables can be represented in the form of additional states in $\mathcal{S}$, making the multi-state models serve as a joint model of the treatment and outcome processes (Andersen and Keiding, 2002). See e.g. Keiding et al (2001) and Gran et al (2008) for examples of such multistate models. A more straightforward way of analysing the effects of such time-dependent treatments would then be to use simulations or so-called gcomputation on this system, as suggested in Keiding et al (2001) and Gran et al (2015). 
2.3 The landmark Aalen-Johansen estimator

The landmark Aalen-Johansen estimator, as suggested by Putter and Spitoni (2018), is given by

$$
\widehat{P}_{l k}^{\mathrm{LMAJ}}(s, t)=\widehat{\boldsymbol{\pi}}^{(\mathrm{LM})}(s) \prod_{s<u \leq t}\left(\mathbf{I}+\Delta \widehat{\boldsymbol{A}}^{(\mathrm{LM})}(u)\right),
$$

for landmark state $l$ and $k \in \mathcal{S} . \widehat{\mathbf{A}}^{(\mathrm{LM})}(t)$ is a matrix of Nelson-Aalen estimates for the cumulative transition intensities, estimated from the landmark data consisting of only the individuals present in state $l$ at time $s . \widehat{\pi}^{(\mathrm{LM})}(s)$ is a $1 \times S$ vector with $l$ 'th element, $\widehat{\pi}_{l}^{(\mathrm{LM})}(s)$ equal to 1 and other elements 0 . Putter and Spitoni prove consistency of $\widehat{P}_{l k}^{\mathrm{LMAJ}}(s, t)$ under the same assumptions required for consistency of AJ estimates of occupation probabilities. This is a consequence of the Datta-Satten result (Datta and Satten, 2001) and the fact that state transition probabilities can be seen as state occupation probabilities for a subsample of the full data or a so-called landmark data set. Similar to the AJ estimator, standard errors for LMAJ estimates can be based on a Greenwood type estimator (Glidden, 2002).

\subsubsection{Landmarking to estimate covariate specific transition probabilities}

By a similar argument as in Section 2.2, the estimator in (3) can be generalised to

$$
\widehat{P}_{l k}^{\mathrm{LMAJ}}(s, t \mid \mathbf{z})=\widehat{\boldsymbol{\pi}}^{(\mathrm{LM})}(s \mid \mathbf{z}) \prod_{s<u \leq t}\left(\mathbf{I}+\Delta \widehat{\boldsymbol{A}}^{(\mathrm{LM})}(u \mid \mathbf{z})\right),
$$

where $\widehat{\boldsymbol{\pi}}^{(\mathrm{LM})}(s \mid \mathbf{z})$ is a vector with element $\widehat{\pi}_{l}^{(\mathrm{LM})}(s \mid \mathbf{z}) \equiv 1$, for any $\boldsymbol{z}$, and zero otherwise. $\widehat{\boldsymbol{A}}^{(\mathrm{LM})}(u \mid \mathbf{z})$ can be estimated by either Cox or Aalen hazard regression based on the landmark data.

\subsubsection{Inverse probability weighted landmarking to estimate transition probabilities}

Following the arguments in Section 2.2, a landmark approach to estimating transition probabilities using inverse probability weighted data, will either reduce to the estimator in (4), with only one covariate (treatment $a$ ), or even further, to the estimator in Equation 3, if the treatment variable is binary and one performs separate analyses for the subsets of weighted data with $a=1$ and $a=0$. Confidence intervals must however be estimated using robust methods or bootstrap, as discussed in Section 2.2.

When the treatment variable $a$ is binary, taking on the values 0 and 1 ; we might fit the logistic regression model

$$
\operatorname{logit} \operatorname{Pr}(a \mid \mathbf{c})=\boldsymbol{\beta}^{T} \mathbf{c}
$$


for the weights. Using this model, it is simple to estimate $\operatorname{Pr}\left(a_{i}=1 \mid \mathbf{c}_{i}\right)$, the conditional probabilities of exposure $a_{i}=1$, for all individuals $i=1, \ldots, n$ given their covariates $\mathbf{c}_{i}$. This probability is often called the propensity score. Individuals with exposure $a_{i}=1$ are assigned the IPT weight corresponding to $w_{i}=1 / \operatorname{Pr}\left(a_{i}=1 \mid \mathbf{c}_{i}\right)$, while individuals with exposure $a_{i}=0$ receive the weight corresponding to $w_{i}=1 /\left(1-\operatorname{Pr}\left(a_{i}=1 \mid \mathbf{c}_{i}\right)\right)$. One can also use stabilized weights (Robins et al, 2000), corresponding to

$$
w_{i}=\frac{\operatorname{Pr}\left(a_{i}=1\right)}{\operatorname{Pr}\left(a_{i}=1 \mid \mathbf{c}_{i}\right)}
$$

when individual $i$ is treated, and

$$
w_{i}=\frac{1-\operatorname{Pr}\left(a_{i}=1\right)}{1-\operatorname{Pr}\left(a_{i}=1 \mid \mathbf{c}_{i}\right)}
$$

when individual $i$ is untreated.

In the weighted data set, covariates are balanced so they are equally represented in both exposure groups. The aim is to mimic a scenario where exposure is independent of covariates. In other words, if the model for the weights is correctly specified, exposure can be seen as randomised in the weighted data set. This is equivalent to comparing a scenario where everyone was exposed to a scenario where no one was exposed. Given the three common causal assumptions (Hernan and Robins, 2018), the weighting procedure removes confounder bias when estimating transition probabilities in observational studies.

\section{A simulation study}

Let us now consider a simulation study to verify the consistency of the LMAJ estimator for transition probabilities in a non-Markov model with covariates. This is done for estimation of transition intensities based on both Cox regression and IPT weighting. We simulate 10000 individual multi-state trajectories by first drawing initial states, baseline covariates and then consecutive transition times between a set of predefined states. Our simulation model has four states; states 1-3 are non-absorbing and interconnected, while state 4 is absorbing and accessible from all other states as illustrated in Figure 1.

Every transition from state $l$ to state $k$ is associated with an intensity $\alpha_{l k}\left(t \mid a, \boldsymbol{c}, \mathcal{H}_{t-}\right)$. Exposure $a$ has two levels, and the covariates $\boldsymbol{c}$ consists of a dichotomous variable $c_{1}$ and a continuous, exponentially distributed, variable $c_{2}$. Both covariates affect the probability of exposure, in addition to transition intensities. The simulation was set up in such a way that transitions into state 3 and 4 from state 1 , and into state 4 from state 3 , were more likely the more time previously spent in state 3 . The opposite was true for transitions from state 3 into state 1 . See supplementary material for more details on data generating algorithm and the corresponding $\mathrm{R}$ code.

For intensities that follow a Cox proportional hazards model, transition times can be generated by giving a parametric specification of the baseline 


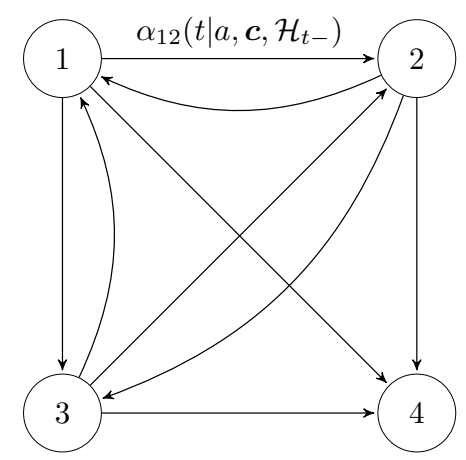

Fig. 1 A multi-state model for simulation. The time dependent transition intensity between state 1 and $2, \alpha_{12}\left(t \mid a, \mathbf{c}, \mathcal{H}_{t-}\right)$, depends on exposure $a$, other covariates $c$ and history up to just before time $t, \mathcal{H}_{t-}$. Corresponding intensities are defined for all transitions, but not shown.

hazard (Bender et al, 2005). Using a Weibull specification, the transition intensities become

$$
\alpha_{l k}\left(t \mid a, \mathbf{c}, \mathcal{H}_{t-}\right)=\lambda_{l k}\left(t \mid \mathcal{H}_{t-}\right) \exp \left(\beta_{l k} a+\theta_{l k}^{T} \mathbf{c}\right) \nu_{l k} t^{\nu_{l k}-1}
$$

The function $\lambda_{l k}\left(t \mid \mathcal{H}_{t-}\right)$ influences the mean transition time and depends on the past history of the transition and at-risk processes. For instance, some transition intensities may depend on the number of earlier stays in specific states or the length of certain stays, which violates the Markov assumption. $\nu_{l k}$ influences both the mean and the shape of the distribution of transition times $\left(\nu_{l k}=1\right.$ equals exponential distribution). The data generating algorithm can be summarised by the following: for $n$ subjects we sample baseline covariates c, exposure $a$ conditional on $\mathbf{c}$, start state $X(0)=l$ from the states $l \in\{1,2,3\}$ and set the censoring time $T_{c}=1000$. From the start state, potential sojourn times for every possible transition $j \neq l$ is generated. Let $T_{i}$ be the minimum of the potential sojourn times. The transition corresponding to the sojourn time $T_{i}$ is then chosen. We repeat this procedure as long as $\sum_{i} T_{i}<T_{c}$ and a transition into state 4 has yet to occur.

With our method for simulating non-Markov multi-state data, there are no simple parameters representing the "true" transition probabilities to which we can compare our estimates. Therefore, we create another data set by simulating similar multi-state data as above, but for a large hypothetical cohort $(n=50$ 000) using fixed covariate values and/or exposure level, and then compare empirical distributions from this data with the estimation results from the main simulation. 

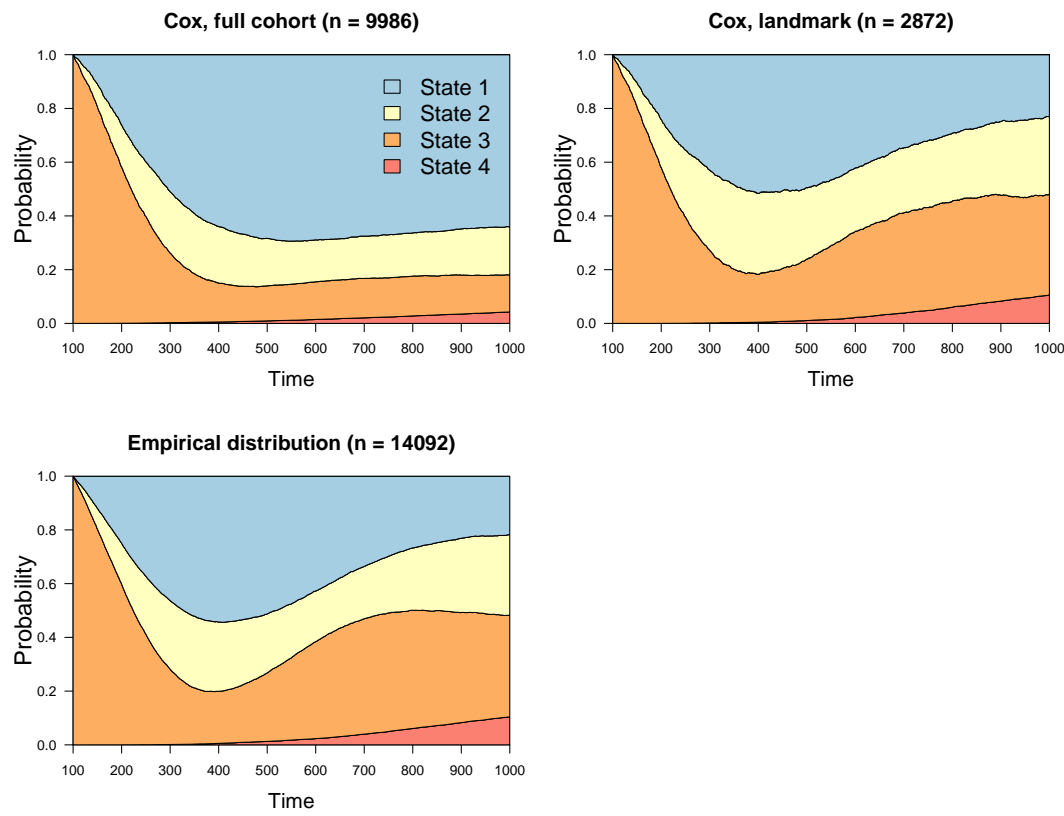

Fig. 2 Estimated conditional state transition probabilities based on Cox regression for the full data set (upper left panel), Cox regression on the landmark sample (upper right panel) and the true empirical distribution (lower left panel).

\subsection{Covariate adjusted analysis}

Consider now a simulated multi-state data set as described above. The data were analysed by estimating conditional transition intensities using Cox proportional hazards models, where we adjusted for exposure and the two other covariates. Based on the estimated intensities, we calculated transition probabilities for going from state 3 at time $s=100$, for a given exposure $a$ and covariate values, $\mathbf{c}$. More specifically, $a=1, c_{1}=1$ and $c_{2}=2$. We calculated both the LMAJ estimate and the traditional AJ estimate. To assess the results, we use a separate simulated data set where all subjects $(n=50000)$ were given the same exposure and covariate values as above. From this data set, we calculate the empirical state distribution at each time-point $(t \geq 100)$, of subjects observed in state 3 at $s=100$. The estimated state transition probabilities are displayed in Figure 2. We see that the landmark estimates converge towards the true (empirical) state transition probability distribution, while the traditional estimate, that relies on the Markov assumption, is heavily biased. 

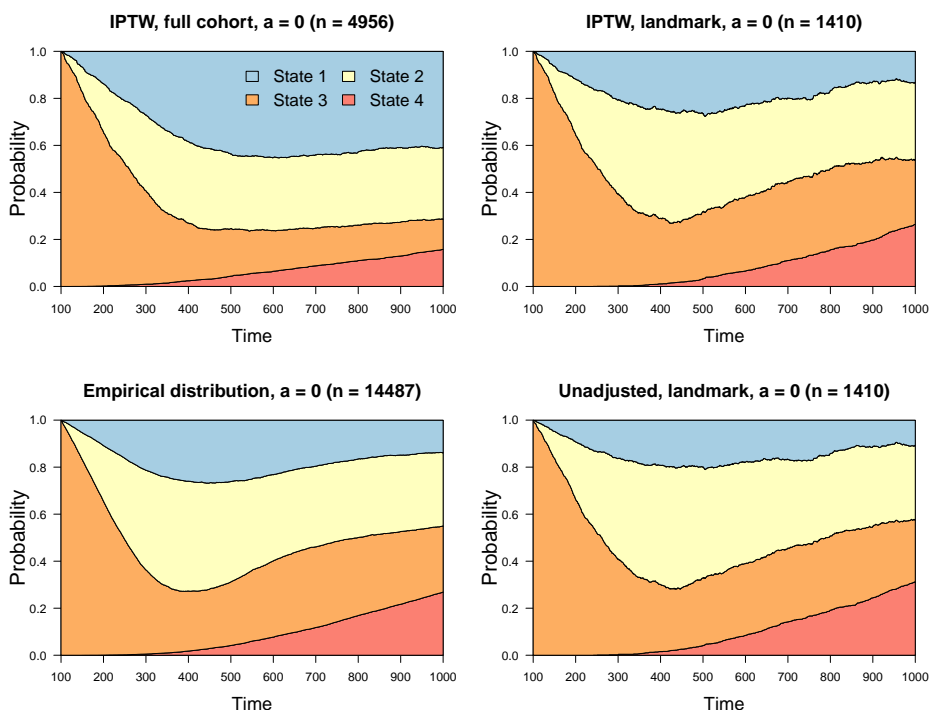

Fig. 3 Estimated state transition probabilities from state 3 based on the inverse probability of treatment weighted Aalen-Johansen estimator (upper left panel), the weighted landmark Aalen-Johansen (upper right panel), the true empirical distribution (lower left panel) and the unweighted landmark Aalen-Johansen (lower right panel).

3.2 Inverse probability of treatment weighted analysis

Let us now consider the same simulated data as before. However, to estimate transition probabilities, we now use IPT weighted LMAJ and AJ estimators, and a marginal unweighted LMAJ for comparison. To simulate the "true" state transition probability distribution, we again used a separate simulated data set where all subjects $(n=50000)$ were given the same exposure regardless of covariate values. From this data set, the empirical state distribution for each time-point $(t \geq 100)$, of subjects observed in state 3 at $s=100$, was calculated. The estimated transition probabilities are shown in Figure 3.

Again, the traditional AJ approach is clearly biased compared to the true empirical results. However, the consequences of failing to perform a weighted analysis (IPTW) are not so clear from this figure. In Figure 4 we have therefore simulated from an identical setup, but with three different sample sizes $n$ and focus on the transition probabilities from state 3 to state 1 . The transition probability is clearly underestimated in the unweighted case. 


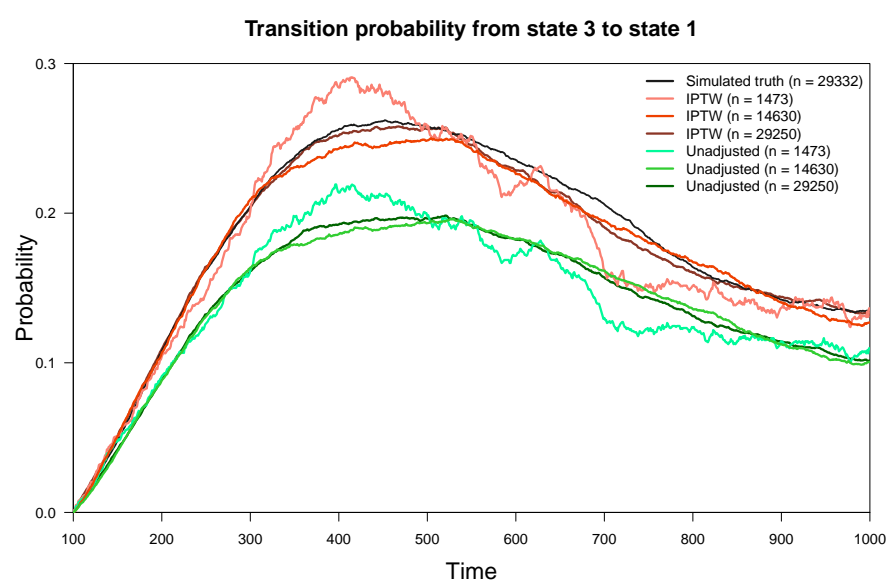

Fig. 4 Transition probabilities from state 3 to state $1, P_{31}(100, t)$, simulated for three different sample sizes $n$. Inverse probability of treatment weighted landmark Aalen-Johansen (LMAJ) estimates (red) are compared with unweighted LMAJ (green) and the true empirical transition probability (black).

\section{An application to a multi-state model for work, education and health-related absence}

To further illustrate the LMAJ estimator, and corresponding estimators after covariate adjustment and weighting, using a real data set, we consider a multi-state model for work, education and health-related absence for young men between the age 21-35. The model, shown in Figure 5, consists of five different states; (1) work, (2) unemployment, (3) sick leave, (4) education (above high school) and (5) disability, where disability is considered an absorbing state. Direct transition from work and education to disability is not allowed by regulations. The model has been analysed before, with the purpose of estimating the effect of high school completion within the age of 23 , but then only with regards to state occupation probabilities (Hoff et al, 2018).

The data consists of information on all males born in Norway between 1971 and 1976 who attended general studies at high school $(n=52128)$. Completing the general studies track leads to admission rights into higher education. Students usually start high school the autumn the year they turn 16 and, if following a normal progression, graduate in spring nearly 3 years later. Those who do not complete within a normal time frame, have the possibility to complete at a later time point. To assess the effect of completion within the age of 21 , the follow-up period was defined to go from July 1st the year students turned 21 (1992 - 1997) and until December 31st 2006 - 2011 (14.5 years). In this period, notifications in national registries of employment, education and welfare benefits were used to construct individual multi-state trajectories. 


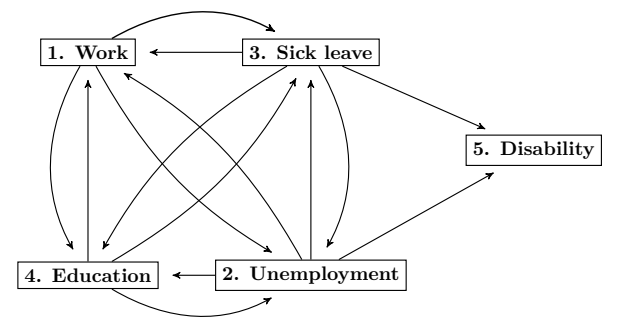

Fig. 5 A multi-state model for work, education and health-related absence.

We also had access to several baseline covariates on an individual level from national registries. These will be described in Sections 4.2 and 4.3 below.

There are several potential problems with assuming that the model in Figure 5 is Markov when analysing these data. For example, it is likely that subjects who have been in work for a longer period have more stable positions than newly employed. Similarly, individuals who have been sick for an extended period, are likely to have lower probability for exiting sick leave from a given time-point than someone who just recently became ill. There are also regulations limiting the duration of the different welfare benefits. In the sections that follow, we will compare results from the landmark approaches for estimating state transition probabilities, allowing for non-Markov data, with the traditional Markov-based approach.

\subsection{Unadjusted analysis}

Let us consider a landmark subsample of individuals on sick leave. The landmark time is an arbitrary autumn time-point five years after inclusion July 1st (five years and 100 days after). We start by comparing (cumulative) transition intensities in the landmark subsample with the full data, shown in Figure 6. Note that we here analyse high school completers and non-completers together. Judging by the estimated transition intensities, it is clear that the landmark subsample differs considerably from the full cohort in terms of transition intensities, which indicates that the Markov assumption is not valid, but there are a few transitions where intensities are more similar, indicating Markov properties for those transitions. As we see in the upper left panel of Figure 6, average stay in other states before transitioning to work is much longer (lower transition intensity) in the landmark subsample compared to the full cohort. For transitions to unemployment (upper right panel), transition intensities are more similar for the landmark subsample and the full cohort, apart from for work to unemployment where it is nearly doubled in the landmark sample. Large differences in transitions intensities can be seen for the remaining transitions.

Corresponding transition probabilities from sick leave to work, as estimated by the LMAJ estimator and the traditional AJ estimator, together with $95 \%$ 
Transitions to work

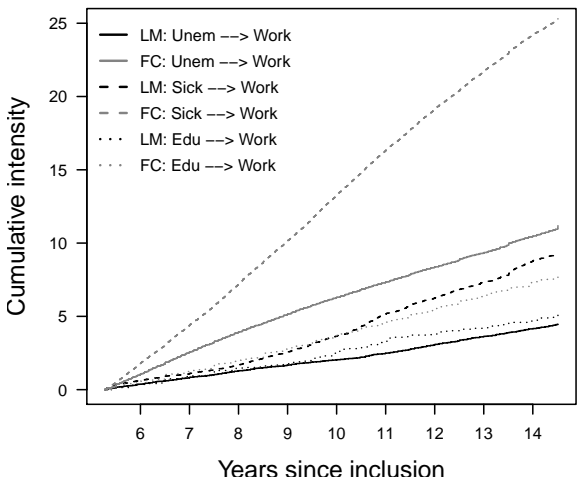

Transitions to sick leave

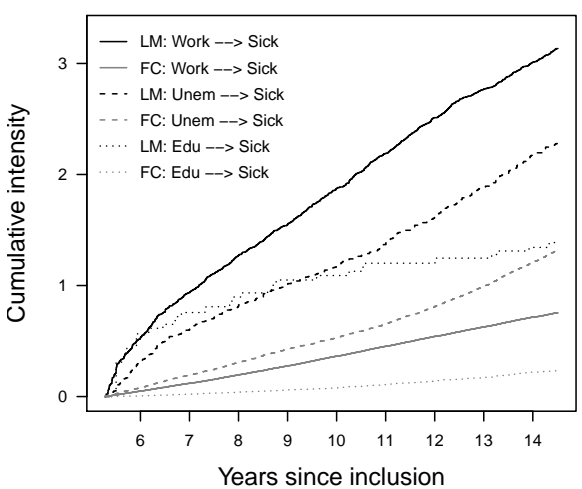

Transitions to disability

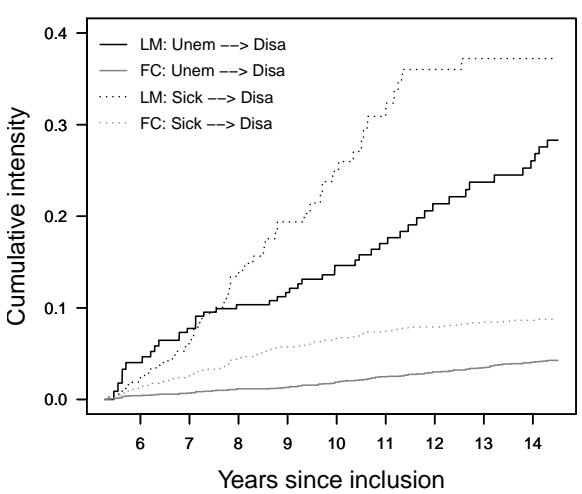

Transitions to unemployment

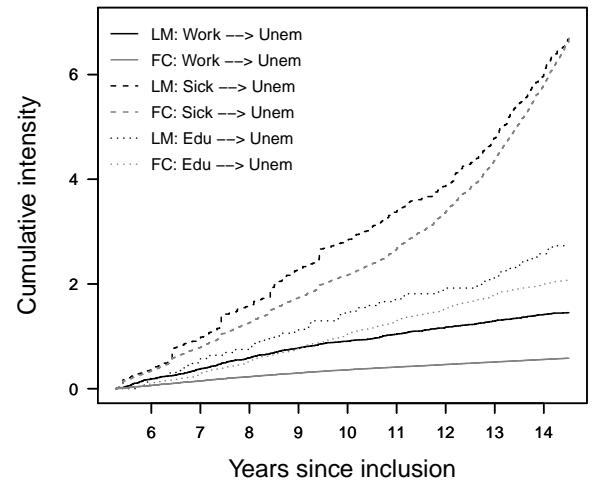

Transitions to education

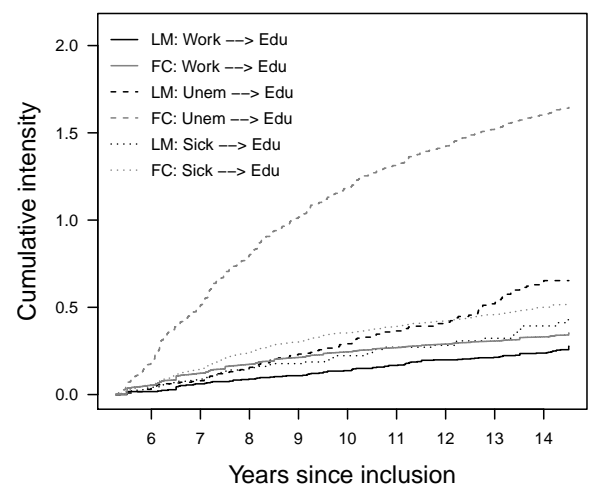

Fig. 6 Cumulative transition intensities starting at the landmark time-point, 5 years and 100 days after original study start. Full drawn lines are Nelson-Aalen estimates based on the landmark sample of individuals starting on sick leave (LM), while dotted lines are traditional Nelson-Aalen estimates based on the full cohort (FC). 

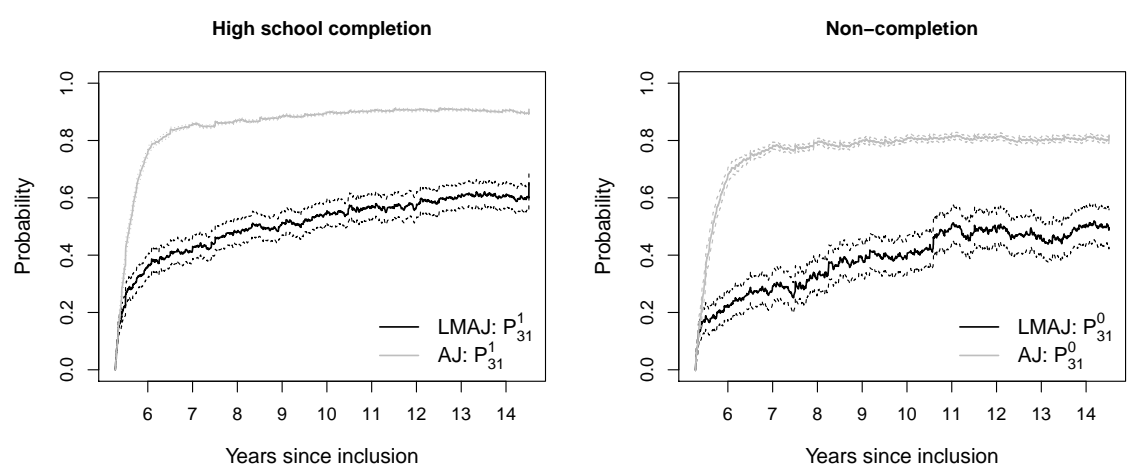

Fig. 7 Transition probabilities from sick leave (state 3) to work (state 1), for high school completers and non-completers respectively, as estimated by Landmark Aalen-Johansen (LMAJ) and traditional Aalen-Johansen (AJ) estimators.

pointwise confidence intervals based on the Greenwood formula, are shown in Figure 7. See also Figure A.1 in online supplementary material for estimated transition probabilities from sick leave to all the four other states. The size of the full cohort and the landmark subsample differ substantially, with 52128 versus 871 individuals, which is reflected in wider confidence intervals for the LMAJ estimates.

The difference in transition probabilities between high school completers and non-completers, $\operatorname{Pr}(X(t)=1 \mid X(s)=3, a=1)-\operatorname{Pr}(X(t)=1 \mid X(s)=$ $3, a=0)$, based on the LMAJ estimator is shown in the left panel of Figure 8. Here $a=1$ corresponds to high school completion and $a=0$ to noncompletion. Also included in the plot are $95 \%$ pointwise confidence intervals. The transition probability from sick leave to work increases about 10 percentage points more for high school completers than non-completers in the months after the landmark time-point and this difference seems to remain throughout the rest of the follow-up period.

\subsection{Covariate adjusted analysis}

Let us now consider the use of Cox regression together with the LMAJ estimator, in order to estimate covariate dependent transition probabilities. Cox proportional hazards models were fitted for all transitions jointly, using the stratified Cox regression approach suggested by de Wreede et al (2011). The models were fitted to the landmark sample with a dichotomous exposure variable for completion versus non-completion and a categorical variable based on IQ test scores. Test scores are registered during Norwegian military conscript examinations and available to us from The Armed Forces Personnel Data Base. The scores are recorded on the standard nine scale (Stanine)(Sundet et al, 2004) ranging from 1 to 9 . A test score of 1 indicates an IQ score below 

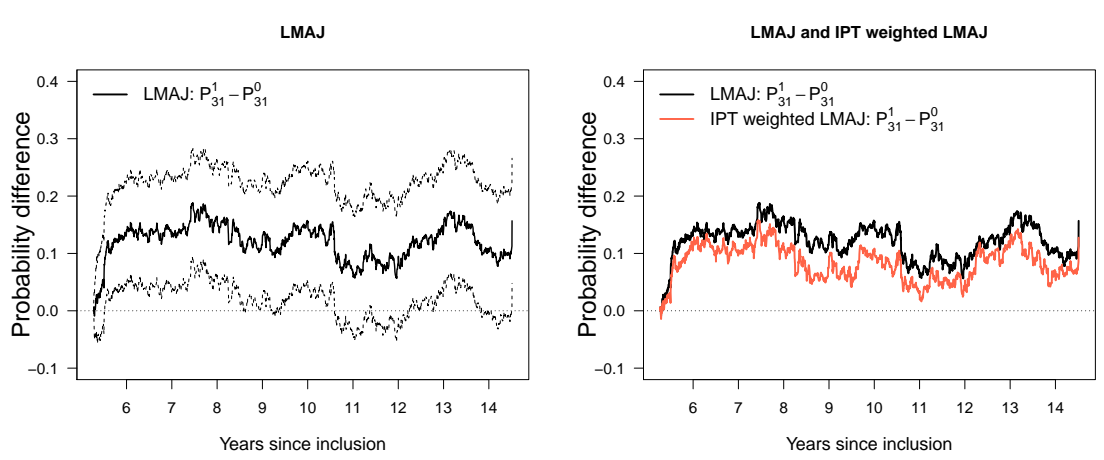

Fig. 8 The effect of high school completion as the difference in transition probabilities from sick leave (state 3 ) to work (state 1), for high school completers versus non-completers. Unadjusted landmark Aalen-Johansen (LMAJ) estimated with $95 \%$ confidence intervals (left panel) and comparison of unadjusted LMAJ estimates and inverse probability of treatment (IPT) weight adjusted LMAJ estimates (right panel).

74 on the traditional Wechsler scale (Wechsler, 1955), 5 is between 96 and 104 while 9 is above 126 . We divided the IQ test scores into three levels; 1-3 (low), 4-6 (middle) and 7-9 (high), which we adjusted for in Cox models for every transition. Using the estimates from the Cox regression as suggested in Section 2.1, we calculated cumulative transition intensities separately for all combinations of exposure and IQ level, six in total. These were then plugged into the matrix product formula. In Figure 9, we can see how the transition probabilities from sick leave to work are higher with higher IQ level and for high school completers compared to non-completers. The two highest IQ levels, 4-6 and 7-9, exhibit coinciding transition probabilities, while they are more reduced for the lowest level 1-3.

\subsection{Inverse probability of treatment weighted analysis}

In addition to IQ scores, we had access to several other baseline covariates, including BMI, military eligibility (mental and physical health) evaluation, parental education level, parental income, maternal marital status, parental disability history, history of childhood chronic disease, regional unemployment rate, year of birth and maternal age at birth $(\leq$ or $>35)$. The data come from several Norwegian population-wide registries. Personal identification numbers allowed for linking within study subjects and between subjects and parents across several registries: Statistics Norway's events database on employment and welfare, FD-Trygd, The National Education Database, The Armed Forces Personnel Data Base and the registries of the Norwegian Labour and Welfare Administration. For a further description of these covariates see Hoff et al (2018). 
High school completion

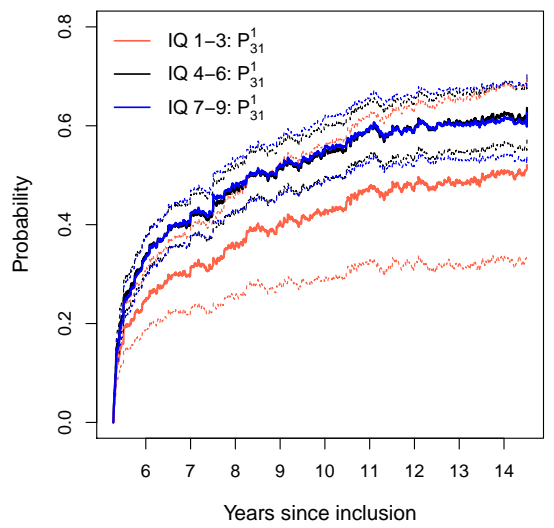

Non-completion

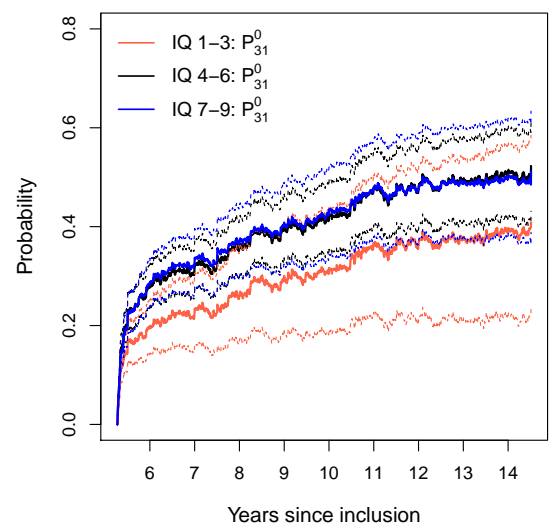

Fig. 9 Estimated transition probabilities from sick leave (state 3) to work (state 1) for high school completers (left panel) and non-completers (right panel), for different levels of IQ; 1-3 (low), 4-6 (middle) and 7-9 (high). Dotted lines are 95\% confidence intervals.

The covariates mentioned above are thought to be confounders between the exposure and outcomes (state transitions). So to make the comparison of completion and non-completion fair, we wanted to adjust for all covariates. Using regression to achieve this, estimated transition probabilities are conditional on covariates being fixed at specific values, but the overall effects of these covariates can be difficult to summarise in a multi-state setting. Therefore, we estimated the marginal effect of high school completion using IPT weighting. Here, the weights mimic a scenario where we can compare everyone, regardless of covariates, completing high school with the situation where no one completed. First, we modelled high school completion using logistic regression, with the mentioned covariates as explanatory variables. Then we used this model to calculate each individual's probability of either completion or non-completion according to what was observed for a given individual. Lastly, each individual was assigned a weight that is the inverse of this probability. The weighted data set was analysed separately for each exposure group by performing weighted Nelson-Aalen estimation of transition intensities. IPTW estimates for transition probability differences are included in the right panel of Figure 8, together with the corresponding unadjusted results, and we see that the weighting reduces the estimated transition probability differences with several percentage points.

\section{Discussion}

In this paper, we have studied the landmark Aalen-Johansen estimator for transition probabilities under deviations from the Markov assumption. The use 
of regression models and inverse probability weights when modelling transition intensities, allowed us to adjust for covariates in the analyses, and simulation studies confirmed that the landmark approach was still consistent.

Our multi-state model for work, education and health-related absence clearly demonstrated deviations from the Markov property. It is reasonable to believe that this would remain a problem with these types of data, even if one tries to partition the state space even further. Transitions from states that are very heterogeneous, in the sense that some individuals are characterised by completely different transition intensities than other types of individuals, are prone to have non-Markov properties. For example, a pooled state with all types of sick leave would exhibit such heterogeneity. Some individuals experience long stays with possibly chronic illness, while others make a short visit with less severe conditions. It is reasonable to assume that individuals on longer visits are overrepresented in a landmark sample from a heterogeneous state. In the traditional AJ estimator, subjects not on sick leave at time $s$, who transition into sick leave after the landmark time-point, will enter the risk set and thereby contribute to estimation. From the cumulative transition intensities plots in Figure 6, it is clear that the population as a whole is much more likely to have short-term visits in sick leave than the landmark subset.

The IPT weights mimic a scenario where the exposure is independent of the included covariates. This is achieved by rebalancing the covariates in the two groups, completion and non-completion. If these covariates account for all confounding factors, this corresponds to a scenario where the exposure is randomised in the target population. Since the landmark analyses in Section 4.3 were conditioned on being on sick leave at time $s$, we can assume that the two exposure groups are more balanced in terms of covariates in the landmark sample than is the case for the full cohort. The more evenly distributed the covariate values are across exposures, the less impact weighting will have on the results.

Using the LMAJ approach, we do not have to assume that the model is Markov to estimate transition probabilities. At the price of having lower precision, we obtain consistent estimates. This approach therefore typically requires data sets with large sample size. In multi-state models with only minor deviances from the Markov property, the higher uncertainty of the landmark estimator could make the standard method preferable (Putter and Spitoni, 2018).

The concept of landmarking could potentially be extended to allow estimation of transition probabilities under even stricter conditioning; not only conditioning on a specific state at time $s$, but also on the further past, such as previous number of visits or days spent in a specific state. Any such conditioning would, as before, come with the cost of decreased power and a need for even larger data sources. However, population-wide registry data, such as the data analysed in this paper, is a good example of data where such approaches are highly favourable. 
Acknowledgements This work was financially supported by the Research Council of Norway (grant no. 237831, 218368 and 273674).

\section{References}

Aalen OO, Borgan Ø, Fekjær H (2001) Covariate adjustment of event histories estimated from Markov chains: the additive approach. Biometrics 57(4):993-1001

Aalen OO, Borgan $\varnothing$, Gjessing H (2008) Survival and event history analysis: a process point of view. Springer, New York, NY

Allignol A, Beyersmann J, Gerds T, Latouche A (2014) A competing risks approach for nonparametric estimation of transition probabilities in a non-Markov illness-death model. Lifetime Data Analysis 20(4):495-513

Andersen PK, Keiding N (2002) Multi-state models for event history analysis. Statistical Methods in Medical Research 11(2):91-115

Andersen PK, Pohar Perme M (2008) Inference for outcome probabilities in multi-state models. Lifetime Data Analysis 14(4):405-431

Andersen PK, Pohar Perme M (2013) Multistate models. In: Klein, JP and van Houwelingen, HC and Ibrahim, JG and Scheike, TH (ed) Handbook of Survival Analysis, Chapman \& Hall/CRC, Boca Raton, FL, pp 417-439

Andersen PK, Borgan $\varnothing$, Gill RD, Keiding N (1993) Statistical models based on counting processes. Springer, New York, NY

Bender R, Augustin T, Blettner M (2005) Generating survival times to simulate Cox proportional hazards models. Statistics in Medicine 24(11):1713-1723

Breslow NE (1972) Discussion of Professor Cox's paper. Journal of the Royal Statistical Society: Series B 34:216-217

Cole SR, Hernán MA, Anastos K, Jamieson BD, Robins JM (2007) Determining the effect of highly active antiretroviral therapy on changes in human immunodeficiency virus type 1 RNA viral load using a marginal structural left-censored mean model. American Journal of Epidemiology 166(2):219-227

Datta S, Satten GA (2001) Validity of the Aalen-Johansen estimators of stage occupation probabilities and Nelson-Aalen estimators of integrated transition hazards for nonMarkov models. Statistics \& Probability Letters 55(4):403-411

Datta S, Satten GA (2002) Estimation of integrated transition hazards and stage occupation probabilities for non-Markov systems under dependent censoring. Biometrics 58(4):792802

Glidden DV (2002) Robust inference for event probabilities with non-Markov event data. Biometrics 58(2):361-368

Gran JM, Wasmuth L, Amundsen EJ, Lindqvist BH, Aalen OO (2008) Growth rates in epidemic models: application to a model for HIV/AIDS progression. Statistics in Medicine $27(23): 4817-4834$

Gran JM, Lie SA, Øyeflaten I, Borgan Ø, Aalen OO (2015) Causal inference in multi-state models-sickness absence and work for 1145 participants after work rehabilitation. BMC Public Health 15(1):1082

Hernan M, Robins JM (2018) Causal Inference. Chapman \& Hall/CRC, Boca Raton

Hoff R, Corbett K, Mehlum IS, Mohn FA, Kristensen P, Hanvold TN, Gran JM (2018) The impact of high-school completion - a multi-state model for work, education and health in young men. Submitted manuscript

Hougaard P (1999) Multi-state models: a review. Lifetime Data Analysis 5(3):239-264

Keiding N, Klein JP, Horowitz MM (2001) Multi-state models and outcome prediction in bone marrow transplantation. Statistics in Medicine 20(12):1871-1885

Lin DY, Wei LJ (1989) The robust inference for the Cox proportional hazards model. Journal of the American Statistical Association 84(408):1074-1078

Meira-Machado LF, de Uña-Álvarez J, Cadarso-Suárez C, Andersen PK (2008) Multi-state models for the analysis of time-to-event data. Statistical Methods in Medical Research 18(2):195-222 
Putter H, Spitoni C (2018) Non-parametric estimation of transition probabilities in nonMarkov multi-state models: The landmark Aalen-Johansen estimator. Statistical methods in medical research 27(7):2081-2092

Putter H, Fiocco M, Geskus RB (2007) Tutorial in biostatistics: competing risks and multistate models. Statistics in Medicine 26(11):2389-2430

Robins JM, Hernan MA, Brumback B (2000) Marginal structural models and causal inference in epidemiology. Epidemiology 11(5):550-560

Rosenbaum PR (1987) Model-based direct adjustment. Journal of the American Statistical Association 82(398):387-394

Røysland K (2011) A martingale approach to continuous-time marginal structural models. Bernoulli 17(3):895-915

Scheike TH (2002) The additive nonparametric and semiparametric Aalen model as the rate function for a counting process. Lifetime Data Analysis 8(3):247-262

Sundet JM, Barlaug DG, Torjussen TM (2004) The end of the Flynn effect?: A study of secular trends in mean intelligence test scores of Norwegian conscripts during half a century. Intelligence 32(4):349-362

Titman AC (2015) Transition probability estimates for non-Markov multi-state models. Biometrics 71(4):1034-1041

de Uña-Álvarez J, Meira-Machado L (2015) Nonparametric estimation of transition probabilities in the non-Markov illness-death model: A comparative study. Biometrics 71(2):364375

Wechsler D (1955) Manual for the wechsler adult intelligence scale.

de Wreede LC, Fiocco M, Putter H (2011) mstate: an R package for the analysis of competing risks and multi-state models. Journal of Statistical Software 38 